\title{
On the Density of Packings of Spheres in Spherical 3-Space*
}

\author{
By
}

\author{
August Florian \\ (Vorgelegt in der Sitzung der math.-nat. Klasse am 13. Oktober 2005 \\ durch das w. M. August Florian)
}

\begin{abstract}
In an $n$-dimensional Euclidean, spherical or hyperbolic space consider a packing of at least four spheres with given radius $r$. The well-known simplicial density bound $d_{n}(r)$ gives an upper bound to the density of such a packing. In spherical 3-space there are exactly four packings for which the density $d_{3}(r)$ is attained. These packings are formed by the inspheres of the regular tilings of type $\{a, 3,3\}$, for $a=2,3,4$ and 5 . In this paper it is proved that $d_{3}$ is a strictly decreasing function of $r$. This implies the existence of the upper bound 0.77963 ... to the density of any packing of at least four congruent spheres in $S^{3}$.
\end{abstract}

Mathematics Subject Classification (2000): 52C17, 05B40.

Key words: Spherical space, packing of spheres, density.

\section{Introduction}

We shall use $S^{n}, E^{n}$ and $H^{n}$ to denote the $n$-dimensional spherical, Euclidean and hyperbolic space, respectively. The space $S^{n}$ can be viewed as the surface of the $(n+1)$-dimensional unit sphere in $E^{n+1}$.

A set of open spheres is said to form a packing in $S^{n}, E^{n}$ or $H^{n}$ if each point of the space belongs to at most one sphere of the set. The most frequently employed method of measuring the efficiency of a

\footnotetext{
* For my brother HELMUT FLORIAN.
} 
packing is to determine its density [6]. As usual, the density of a system of subsets of $S^{n}$ is defined as the ratio of the total $n$-dimensional volume of the sets to the volume of $S^{n}$. The density of a system of bodies in $E^{n}$ is defined, roughly speaking, as the ratio of the total volume of the members of the system to the volume of the whole space. This is made precise by an appropriate limit process. A general definition of the density of an arbitrary arrangement of subsets of $H^{n}$ with respect to the whole space is not available. The difficulties arising in this connection are discussed in some detail in [6]. Thus only statements of a local character can be expected concerning density in $H^{n}$.

In $S^{n}, E^{n}$ or $H^{n}(n \geq 2)$ consider a regular simplex of edge length $2 r$ (in $S^{n}: 2 r<\arccos (-1 / n)$ ), and the system of $n+1$ spheres of radius $r$, with their centres at the vertices of the simplex. The spheres of the system do not overlap. Let $d_{n}(r)$ denote the ratio of the volume of the part of the simplex covered by the spheres to the volume of the whole simplex. In a paper of 1959, L. FEJES TóTH [10] stated the conjecture that in $S^{n}, E^{n}$ or $H^{n}$, each packing of at least $n+1$ spheres of radius $r$ has a density

$$
d \leq d_{n}(r) .
$$

In the case of the hyperbolic space, (1) has to be replaced by an appropriate local version. As is well known, inequality (1) is true for $n=2$; see [8] or [11]. In two earlier papers L. FEJES TóTH [9] and COXETER [5], independently of each other, had put forward conjecture (1) in the case $n=3$. They advanced some arguments to support it and pointed out some interesting consequences, especially for packings in non-Euclidean spaces.

About the time when FEJES TÓTH's conjecture was published, ROGERS $[15,16]$ succeeded in proving (1) for packings in $E^{n}(n \geq 2)$. Since in $E^{n}$ the bound $d_{n}(r)$ does not depend on $r$, we shall simply write $d_{n}$ for $d_{n}(r)$.

A packing of congruent spheres is said to be saturated if any additional sphere of the same size overlaps some member of the packing. Let us consider a saturated packing of $n$-dimensional congruent spheres. To each sphere $S$ of the packing we assign the set $P(S)$ of all points of space, whose distance from the centre of $S$ is not greater than their distance from the centre of any other sphere of the system. The set $P(S)$ is a closed, bounded and convex polytope containing $S$. The polytopes $P(S)$, corresponding to the different spheres of the system, fit together, without overlapping and without gaps, to fill up the whole space; they are called the Voronoi polytopes of the packing. 
The required inequality (1) is proved for $E^{n}$ by showing that, for each sphere $S$ of the packing, the ratio of the volume of $S$ to the volume of the assigned Voronoi polytope $P(S)$ does not exceed $d_{n}$.

Note that BARANOVSKIII [1] independently proved the simplicial bound (1) for $E^{n}$.

In the special case when $n=2$, we have $d_{2}=\pi / \sqrt{12}$. The incircles of the regular tiling $\{6,3\}$ form a packing with maximum density $\pi / \sqrt{12}[11]$.

When $n=3$, inequality (1) shows that the density of each packing of equal spheres in $E^{3}$ satisfies

$$
d \leq d_{3}=\sqrt{18}\left(\arccos \frac{1}{3}-\frac{\pi}{3}\right)=0.77963 \ldots
$$

The dihedral angle of a regular tetrahedron, i.e. $\arccos 1 / 3$, lies between $2 \pi / 6$ and $2 \pi / 5$. Therefore, the density $d_{3}$ cannot be attained by putting together congruent regular tetrahedra to fill up the whole space.

ROGERS' upper bound on the density of packing in $E^{3}$ has been successively reduced. MUDER's result [13]

$$
d \leq 0.77305 \ldots \quad\left(E^{3}\right)
$$

is at present the best completely published upper bound to the density of packings of equal spheres in $E^{3}$.

The review articles [6], [7], [2], [12] contain more information on packing of spheres (also in higher dimensions) and related topics.

Let us now consider packings of equal spheres in spherical and hyperbolic spaces.

Let $M(n, r)$ denote the maximum number of spheres with radius $r<\pi / 2$ which can be placed on $S^{n}$ without overlapping. RANKIN [14] determined the exact values of $M(n, r)$ for all $n \geq 2$ and $r \geq \pi / 4$.

In 1963 BÖRÖCZKY [3] proved FEJES TÓTH's conjecture (1) for $n=3$. More precisely: In $S^{3}, E^{3}$ or $H^{3}$ consider a saturated packing of at least 4 spheres with radius $r$ (on $S^{3}: r<\pi / 4$ ). Then the density of each sphere of the packing with respect to its Voronoi polyhedron is not greater than $d_{3}(r)$.

This formulation does not refer to a concept of global density and thus also applies to hyperbolic space. The statement clearly implies that the density of packing with respect to the whole space $S^{3}$ or $E^{3}$ is also bounded by $d_{3}(r)$. Several years later, BÖRÖCZKY [4] proved FEJES TóTH's conjecture (1) for packings of equal spheres in $n$-dimensional spaces (on $S^{n}: r<\pi / 4$ ). 
Let us return to packings in 3-dimensional spaces, first in $H^{3}$.

In the common paper with BÖRÖCZKY [3], FLORIAN proved that $d_{3}(r)$ is a strictly increasing function for $0<r<\infty$. Thus the (local) density of any packing of equal spheres in $H^{3}$ satisfies

$$
d<\lim _{r \rightarrow \infty} d_{3}(r) .
$$

The elegant representation of the upper bound

$$
\lim _{r \rightarrow \infty} d_{3}(r)=\left(1+\frac{1}{2^{2}}-\frac{1}{4^{2}}-\frac{1}{5^{2}}+\frac{1}{7^{2}}+\frac{1}{8^{2}}--++\right)^{-1}=0.853 \ldots
$$

is due to COXETER [5]. The bound is attained by the packing of horospheres inscribed in the cells of the 3-dimensional regular tiling $\{6,3,3\}$.

Finally let us turn to packings of equal spheres in $S^{3}$ and BÖRÖCZKY's tetrahedral density bound $d_{3}(r)$. It is convenient to consider, in addition to the side-length $2 r$, the dihedral angle of a regular tetrahedron. In $S^{3}$, a non-degenerate regular tetrahedron with dihedral angle $2 \alpha$ exists if and only if

$$
\arccos \frac{1}{3}<2 \alpha<\pi \text {. }
$$

The lower bound is the dihedral angle of a regular tetrahedron in $E^{3}$. When $2 \alpha=\pi$, the tetrahedron $T(2 \alpha)$ degenerates into a half-space of $S^{3}$. The edge-length $2 r$ is related to $2 \alpha$ by the equation

$$
\cos 2 \alpha=\frac{\cos 2 r}{1+2 \cos 2 r}
$$

showing that $\alpha$ is a strictly increasing function of $r$. Inequality (4) is equivalent to

$$
0<2 r<\arccos \left(-\frac{1}{3}\right)=2 \arctan \sqrt{2} .
$$

In this paper we prove the following theorem.

Theorem. In $S^{3}$, the density bound $d_{3}(r)$ is a strictly decreasing function, for $0<r \leq \arctan \sqrt{2}$.

Corollary. The density of any packing of at least 4 equal spheres in $S^{3}$ is less than

$$
\lim _{r \rightarrow 0} d_{3}(r)=\sqrt{18}\left(\arccos \frac{1}{3}-\frac{\pi}{3}\right)=0.77963 \ldots .
$$


On $S^{3}$ there are exactly 4 regular tilings, the cells of which are tetrahedra. They correspond to the dihedral angle $2 \pi / k$, for $k=2,3,4,5$ :

$$
\{3,3,2\}, \quad\{3,3,3\}, \quad\{3,3,4\}, \quad\{3,3,5\} .
$$

The vertices of the tilings are centres of 4, 5, 8 and 120 equal spheres touching each other in each case which form a densest packing of spheres with the respective radius. They are the inspheres of the cells of the dual tilings $\{2,3,3\},\{3,3,3\},\{4,3,3\},\{5,3,3\}$. The following list contains the radii and corresponding densities of packings.

$$
\begin{array}{llll}
\{2,3,3\} & r_{2}=\frac{1}{2} \arccos \left(-\frac{1}{3}\right), & d\left(r_{2}\right)=0.61613 \ldots, \\
\{3,3,3\} & r_{3}=\frac{1}{2} \arccos \left(-\frac{1}{4}\right), & d\left(r_{3}\right)=0.68057 \ldots, \\
\{4,3,3\} & r_{4}=\pi / 4, & d\left(r_{4}\right)=0.72676 \ldots, \\
\{5,3,3\} & r_{5}=\pi / 10, & d\left(r_{5}\right)=0.77412 \ldots
\end{array}
$$

There is no other packing of spheres with radius $r$ and density $d_{3}(r)$, for $0<r \leq \arctan \sqrt{2}$. This follows from BÖRÖCZKY's paper [4] for $0<r<\pi / 4$, and RANKIN [14] for $r=\pi / 4$. The cases when $r>\pi / 4$ can be settled in a direct way, since only 5 or 4 spheres are involved. Combining the Theorem with RANKIN's result [14] for $n=3$ we see that BÖRÖCZKY's density bound $d \leq d_{3}(r)$ holds for $0<r \leq \arctan \sqrt{2}$.

It is remarkable that $d\left(r_{5}\right)$ is greater than the density of any packing of equal spheres in $E^{3}$, see (3). This is in contrast to the corresponding situation in dimension 2 , see [11].

The inspheres of $\{5,3,3\}$ form a densest packing of at least 4 and at most 120 equal spheres. Observe that $d\left(r_{5}\right)$ is rather close to $\lim _{r \rightarrow 0} d_{3}(r)=0.77963 \ldots$. It may be that the inspheres of $\{5,3,3\}$ even form a densest packing of at least 4 equal spheres in $S^{3}$ (see [10]). At present, however, we are far from being able to prove this conjecture.

Observe that the density of the densest packing of spheres with radius $r$ is not decreasing in any subinterval of $0<r<\arctan \sqrt{2}$. Thus, the behaviour of this function is quite different from that of $d_{3}(r)$.

\section{Proof of the Theorem}

Let us recall the definition of the tetrahedral density bound $d_{3}(r)$ in the case of the 3-dimensional spherical space.

Consider a system of 4 spheres of radius $r(0<r \leq \arctan \sqrt{2})$ touching each other. Let $T$ be the tetrahedron with its vertices at the centres of the spheres. Then $d_{3}(r)$ is the ratio of the volume of the 
part of $T$ covered by the spheres of the system to the volume of $T$. The dihedral angle $2 \alpha$ of $T$ is connected with $r$ by the equation

$$
\cos 2 \alpha=\frac{\cos 2 r}{1+2 \cos 2 r} \text {. }
$$

Then

$$
d_{3}(r)=\frac{4(6 \alpha-\pi)}{4 \pi V(T(2 \alpha))} \pi(2 r-\sin 2 r) .
$$

The volume of the tetrahedron $T(2 \alpha)$ follows from Schläfli's differential form by integration

$$
V(T(2 \alpha))=6 \int_{\alpha_{0}}^{\alpha} 2 r(u) d u, \quad \cos 2 r(u)=\frac{\cos 2 u}{1-2 \cos 2 u},
$$

where $2 \alpha_{0}=\arccos (1 / 3)$ is the dihedral angle in $E^{3}$. Formula (9) takes the form

$$
d_{3}(r)=\frac{6 \alpha-\pi}{6 I}(2 r-\sin 2 r)
$$

where

$$
I=\int_{\alpha_{0}}^{\alpha} 2 r(u) d u .
$$

Referring to (8), (10), (11) and (12) we shall prove that

$$
d_{3}^{\prime}(r)<0
$$

for $0<r<\arctan \sqrt{2}$.

For brevity, we write

$$
\tan r=t
$$

so that

$$
0<t<\sqrt{2} .
$$

From (8) and (12) we obtain

$$
\frac{d \alpha}{d r}=\frac{t\left(1+t^{2}\right)}{\sqrt{2-t^{2}}\left(3-t^{2}\right)}, \quad \frac{d I}{d r}=2 r \frac{d \alpha}{d r} .
$$

Here and in the following we omit straightforward calculations. Using (15) we get from (11)

$$
6 I^{2} d_{3}^{\prime}(r)=\frac{24 t^{2}}{1+t^{2}} g(r) f_{1}(r),
$$


where

$$
g(r)=I-\frac{r}{2} \frac{\left(1+t^{2}\right)^{2}}{t \sqrt{2-t^{2}}\left(3-t^{2}\right)}(2 r-\sin 2 r)
$$

and

$$
f_{1}(r)=\frac{\left(1+t^{2}\right)^{2}(2 r-\sin 2 r) I}{4 t \sqrt{2-t^{2}}\left(3-t^{2}\right) g(r)}+\alpha-\frac{\pi}{6} .
$$

Since $2 r>\sin 2 r, t>r$ and $t^{2}<2$, we see that $g^{\prime}(r)=\frac{\left(1+t^{2}\right)^{2}(2 r-\sin 2 r)}{2 t^{2}\left(2-t^{2}\right)^{3 / 2}\left(3-t^{2}\right)^{2}}\left[r\left(6-30 t^{2}+12 t^{4}\right)-t\left(3-t^{2}\right)\left(2-t^{2}\right)\right]<0$.

Because, moreover, $\lim _{r \rightarrow 0} g(r)=0$ we have

$$
g(r)<0
$$

for $0<r<\arctan \sqrt{2}$.

Introducing the abbreviation

$$
\begin{aligned}
4 t \sqrt{2-t^{2}}\left(3-t^{2}\right) g(r) & =4 t \sqrt{2-t^{2}}\left(3-t^{2}\right) I-2 r\left(1+t^{2}\right)^{2}(2 r-\sin 2 r) \\
& =N(r)
\end{aligned}
$$

we obtain from (18)

$$
\frac{N^{2}}{I} f_{1}^{\prime}(r)=\left(1+t^{2}\right) g_{1}(r) f_{2}(r),
$$

where

$$
\begin{aligned}
g_{1}(r)= & \frac{1}{\sqrt{2-t^{2}}}\left[3\left(1+t^{2}\right)\left(-1+5 t^{2}-2 t^{4}\right)(2 r-\sin 2 r)\right. \\
& \left.+4 t^{3}\left(2-t^{2}\right)\left(3-t^{2}\right)\right]
\end{aligned}
$$

and

$$
f_{2}(r)=8 I+2\left(1+t^{2}\right)^{2} \frac{\left(1+t^{2}\right)(2 r-\sin 2 r)-8 t^{2} r}{g_{1}(r)}(2 r-\sin 2 r) .
$$

First we show that

$$
g_{1}(r)>0
$$

for $0<r<\arctan \sqrt{2}$. This is clear if $-1+5 t^{2}-2 t^{4} \geq 0$. Let now $-1+5 t^{2}-2 t^{4}<0$, i.e., $t^{2}<(5-\sqrt{17}) / 4<\frac{1}{4}$. Since $r<t<\sqrt{2}$, we 
have $2 r<2 \sqrt{2}$ and $(2 r)^{2}<8$. Thus

$$
2 r-\sin 2 r=\frac{(2 r)^{3}}{3 !}-\frac{(2 r)^{5}}{5 !}+\cdots<\frac{4}{3} r^{3}
$$

and

$$
3(2 r-\sin 2 r)<4 r^{3}<4 t^{3} .
$$

From

$$
\left|-1+5 t^{2}-2 t^{4}\right|=1-5 t^{2}+2 t^{4}<1+2 t^{4}<\frac{9}{8}
$$

and $1+t^{2}<\frac{5}{4}$ we conclude that

$$
\left|\left(1+t^{2}\right)\left(-1+5 t^{2}-2 t^{4}\right)\right|<\frac{5}{4} \cdot \frac{9}{8}=\frac{45}{32} .
$$

On the other hand we have

$$
\left(2-t^{2}\right)\left(3-t^{2}\right)>\frac{7}{4} \cdot \frac{11}{4}=\frac{77}{16}>\frac{45}{32},
$$

as required.

From (22) it follows that

$$
\lim _{r \rightarrow 0} \frac{g_{1}(r)}{2 r-\sin 2 r}=\frac{15}{\sqrt{2}},
$$

and from (23)

$$
\lim _{r \rightarrow 0} f_{2}(r)=0 .
$$

Differentiation of $f_{2}(r)$ yields in a first step

$$
\begin{aligned}
\frac{g_{1}^{2} f_{2}^{\prime}(r)}{2\left(1+t^{2}\right)^{2}(2 r-\sin 2 r)}= & g_{1} \frac{8 r t}{\left(2-t^{2}\right)\left(3-t^{2}\right)}\left(-3+15 t^{2}-6 t^{4}\right) \\
& +g_{1} \cdot 4 t\left[\left(1+t^{2}\right)(2 r-\sin 2 r)-8 t^{2} r\right] \\
& +g_{1}\left[2 t\left(1+t^{2}\right)(2 r-\sin 2 r)-16 r t\left(1+t^{2}\right)\right] \\
& -\left[\left(1+t^{2}\right)(2 r-\sin 2 r)-8 t^{2} r\right] g_{1}^{\prime}(r),
\end{aligned}
$$

where $g_{1}^{\prime}(r)$ can be calculated from (22),

$$
\begin{aligned}
g_{1}^{\prime}(r)= & \frac{t\left(1+t^{2}\right)}{\left(2-t^{2}\right)^{3 / 2}}\left[\left(1+t^{2}\right)\left(-3+15 t^{2}-6 t^{4}\right)(2 r-\sin 2 r)\right. \\
& \left.+4 t^{3}\left(2-t^{2}\right)\left(3-t^{2}\right)\right] \\
& +\frac{1}{\sqrt{2-t^{2}}}\left[2 t\left(1+t^{2}\right)\left(12+18 t^{2}-18 t^{4}\right)(2 r-\sin 2 r)\right. \\
& \left.+4 t^{2}\left(15+8 t^{2}-24 t^{4}+7 t^{6}\right)\right] .
\end{aligned}
$$


Collecting the terms containing the same power of $2 r-\sin 2 r$, from (26) and (27) we obtain

$$
\frac{g_{1}^{2} f_{2}^{\prime}(r)}{2\left(1+t^{2}\right)^{2}(2 r-\sin 2 r)}=A \cdot(2 r-\sin 2 r)^{2}+B(2 r-\sin 2 r)
$$

with

$$
\begin{aligned}
A= & \frac{t\left(1+t^{2}\right)^{2}}{\left(2-t^{2}\right)^{3 / 2}}\left(-81+138 t^{2}-63 t^{4}+6 t^{6}\right), \\
B= & \frac{4 t^{2}\left(1+t^{2}\right)}{\sqrt{2-t^{2}}}\left(-15+25 t^{2}-8 t^{4}\right) \\
& +\frac{8 r t\left(1+t^{2}\right)}{\left(2-t^{2}\right)^{3 / 2}\left(3-t^{2}\right)}\left(45-57 t^{2}-6 t^{4}+57 t^{6}-33 t^{8}+6 t^{10}\right) .
\end{aligned}
$$

If we replace $\sin 2 r$ by $2 t /\left(1+t^{2}\right)$, it follows from (28) and (29) that

$$
\begin{aligned}
& \frac{g_{1}^{2} f_{2}^{\prime}(r)}{2\left(1+t^{2}\right)^{2}(2 r-\sin 2 r)^{2}} \\
&=\frac{2 t\left(1+t^{2}\right)}{\left(2-t^{2}\right)^{3 / 2}\left(3-t^{2}\right)}\left\{t\left(63-45 t^{2}-49 t^{4}+49 t^{6}-10 t^{8}\right)\right. \\
&\left.+r\left(-63+24 t^{2}+144 t^{4}-18 t^{6}-57 t^{8}+18 t^{10}\right)\right\} \\
&= \frac{2 t\left(1+t^{2}\right)^{2}}{\left(2-t^{2}\right)^{3 / 2}\left(3-t^{2}\right)}\left[t P_{1}(t)+r P_{2}(t)\right] .
\end{aligned}
$$

Here we have used the abbreviations

$$
\begin{aligned}
& P_{1}(t)=-10\left(t^{2}-3\right)\left(t^{2}-1.5\right)\left(t^{2}-1.4\right), \\
& P_{2}(t)=18 t^{8}-75 t^{6}+57 t^{4}+87 t^{2}-63 .
\end{aligned}
$$

First let us assume that

$$
0<t \leq 1 \text {. }
$$

Obviously, $P_{1}(t)>0$, while $P_{2}(t)$ changes from negative to positive values. We shall now show that

$$
t P_{1}(t)+r P_{2}(t)>0 \text {. }
$$

Let $P_{2}(t)<0$. Since

$$
r=\arctan t<t-\frac{t^{3}}{3}+\frac{t^{5}}{5}
$$


we have

$$
\begin{aligned}
t P_{1}(t)+r P_{2}(t) & >t P_{1}(t)+\left(t-\frac{t^{3}}{3}+\frac{t^{5}}{5}\right) P_{2}(t) \\
& =t^{5}\left(3.6 t^{8}-21 t^{6}+54.4 t^{4}-86.6 t^{2}+74.4\right) .
\end{aligned}
$$

It can easily be proved that the last term is positive for $0<t \leq 1$.

Second let us assume that

$$
1 \leq t \leq \sqrt{2}
$$

Observe that, by (31), $P_{1}(t)$ is positive, except for the interval $\sqrt{1.4}<t<\sqrt{1.5}$, where $P_{1}(t)<0$. Because $P_{2}(t)>0$ in the whole interval and

$$
r=\arctan t>t-\frac{t^{3}}{3}
$$

we have

$$
\begin{aligned}
t P_{1}(t)+r P_{2}(t) & >t P_{1}(t)+\left(t-\frac{t^{3}}{3}\right) P_{2}(t) \\
& =t^{5}\left(-6 t^{6}+43 t^{4}-104 t^{2}+87\right) .
\end{aligned}
$$

It can easily be shown that the last term is positive, so that (33) also holds for $1 \leq t \leq \sqrt{2}$.

The last two results combined with (30) imply that

$$
f_{2}^{\prime}(r)>0
$$

for $0<t<\sqrt{2}$. From (35) and (25) it follows that

$$
f_{2}(r)>0 \text {. }
$$

From (36), (24) and (21) we conclude that

$$
f_{1}^{\prime}(r)>0 \text {, }
$$

for $0<r<\arctan \sqrt{2}$.

In order to calculate $\lim _{r \rightarrow 0} f_{1}(r)$ we refer to (18). Making use of (15) we obtain the expansion of $I(r)$ into a power series

$$
I(r)=\frac{1}{3 \sqrt{2}}\left(\frac{2}{3} r^{3}+\cdots\right) .
$$

Hence

$$
(2 r-\sin 2 r) I(r)=\frac{1}{3 \sqrt{2}}\left(\frac{8}{9} r^{6}+\cdots\right) .
$$


On the other hand, we get from (20) and (38)

$$
4 t \sqrt{2-t^{2}}\left(3-t^{2}\right) g(r)=-\frac{12}{5} r^{6}+\cdots .
$$

Then, by (18), (39) and (40) in conjunction with (5),

$$
\lim _{r \rightarrow 0} f_{1}(r)=-\frac{10}{81 \sqrt{2}}+\frac{1}{2} \arccos \frac{1}{3}-\frac{\pi}{6}=0.004583 \ldots>0 .
$$

The combination of (37) and (41) shows that

$$
f_{1}(r)>0
$$

for $0<r<\arctan \sqrt{2}$.

The desired inequality (13) is a consequence of (16), (19) and (42).

\section{Acknowledgement}

I would like to thank Prof. J. LINHART for his careful reading of the paper and for correcting an error.

\section{References}

[1] BARANOVSKIĬ, E. P. (1964) On packing of $n$-dimensional Euclidean spaces by equal spheres (Russian). Izv. Vyš̌. Učebn. Zaved. Matematika 39: 14-24

[2] BEZDEK, K. (to appear) Sphere packings revisited. Europ. J. Comb.

[3] BöRÖCZKY, K., FloRIAN, A. (1964) Über die dichteste Kugelpackung im hyperbolischen Raum. Acta Math. Acad. Sci. Hungar. 15: 237-245

[4] BÖRÖCZKY, K. (1978) Packing of spheres in spaces of constant curvature. Acta Math. Acad. Sci. Hungar. 32: 243-261

[5] CoXeter, H. S. M. (1954) Arrangements of equal spheres in non-Euclidean spaces. Acta Math. Acad. Sci. Hungar. 5: 263-274

[6] Fejes Tóth, G., KuPERBerg, W. (1993) Packing and covering with convex sets. In: Handbook of Convex Geometry (Gruber, P. M., WiLLS, J. M., eds.), pp. 799-860. North-Holland, Amsterdam

[7] Fejes Tóth, G. (2004) Packing and covering. In: Handbook of Discrete and Computational Geometry (GoOdMAN, J. E., et al., eds.), 2nd enlarged Ed., pp. 25-53. CRC Press, Boca Raton

[8] Fejes Tóth, L. (1953) Kreisausfüllungen der hyperbolischen Ebene. Acta Math. Acad. Sci. Hungar. 4: 103-110

[9] Fejes Tóth, L. (1953) On close-packings of spheres in spaces of constant curvature. Publ. Math. Debrecen 3: 158-167

[10] Fejes TóTH, L. (1959) Kugelunterdeckungen und Kugelüberdeckungen in Räumen konstanter Krümmung. Arch. Math. 10: 307-313

[11] Fejes Tóth, L. (1964) Regular Figures. Pergamon Press, Oxford

[12] Florian, A. (to appear) On the density of packings of spheres in spaces of constant curvature. Rend. Circ. Mat. Palermo 
100 A. Florian: On the Density of Packings of Spheres in Spherical 3-Space

[13] Muder, D. J. (1993) A new bound on the local density of sphere packings. Discrete Comput. Geom. 10: 351-375

[14] Rankin, R. A. (1954-1956) The closest packing of spherical caps in $n$ dimensions. Proc. Glasgow Math. Assoc. 2: 139-144

[15] Rogers, C. A. (1958) The packing of equal spheres. Proc. London Math. Soc. 8: 609-620

[16] Rogers, C. A. (1964) Packing and Covering. Cambridge University Press, Cambridge

Author's address: Prof. Dr. August Florian, Department of Mathematics, University of Salzburg, Hellbrunner Straße 34, 5020 Salzburg, Austria. E-Mail: august.florian@sbg.ac.at. 